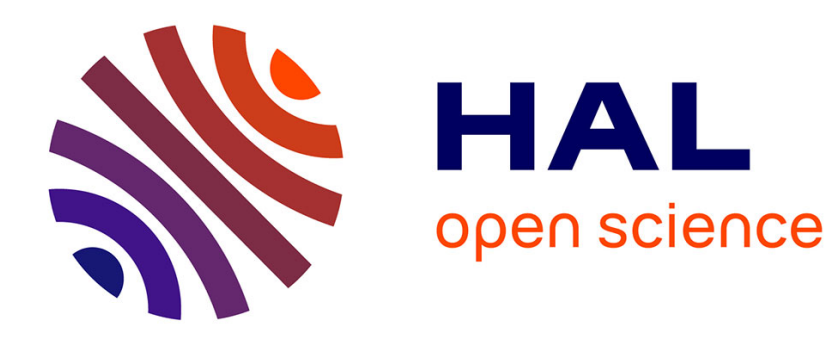

\title{
La planification de séquences d'enseignement, objet de description ou de prescription?
}

\author{
Philippe Dessus
}

\section{To cite this version:}

Philippe Dessus. La planification de séquences d'enseignement, objet de description ou de prescription?. Revue Française de Pédagogie, 2000, 133, pp.101-116. 10.3406/rfp.2000.1024 hal-01233418

\section{HAL Id: hal-01233418 \\ https://hal.univ-grenoble-alpes.fr/hal-01233418}

Submitted on 25 Nov 2015

HAL is a multi-disciplinary open access archive for the deposit and dissemination of scientific research documents, whether they are published or not. The documents may come from teaching and research institutions in France or abroad, or from public or private research centers.
L'archive ouverte pluridisciplinaire HAL, est destinée au dépôt et à la diffusion de documents scientifiques de niveau recherche, publiés ou non, émanant des établissements d'enseignement et de recherche français ou étrangers, des laboratoires publics ou privés. 
Revue Française de Pédagogie, $\mathrm{n}^{\circ}$ 133, 2000, p. 101-116

\title{
LA PLANIFICATION DE SEQUENCES D'ENSEIGNEMENT, OBJET DE DESCRIPTION OU DE PRESCRIPTION?
}

\author{
Philippe DESSUS \\ « Tous nos malheurs proviennent de ce que \\ les hommes ne savent pas ce qu'ils sont, \\ et ne s'accordent pas sur ce qu'ils veulent être. ” \\ D. M. TEMPLEMORE
}

Les chercheurs dans le domaine de la technologie de l'éducation débattent à propos des vues descriptive et prescriptive de leur objet d'étude, respectivement l'activité réellement mise en œuvre et celle qui devrait l'être. Cet article a pour objet de présenter, sous ces deux points de vue, l'activité de planification de séquences d'enseignement, selon les principaux courants ayant étudié cette activité : behaviorisme, technologie des médias, systémique, cognitivisme et constructivisme. Nous détaillons des travaux de ces courants en montrant qu'ils peuvent être classés en quatre catégories, selon la manière dont sont croisés l'origine et l'usage des vues descriptive et prescriptive. Nous montrons que ces deux approches sont toutes deux nécessaires.

Is Instructional Design Descriptive or Prescriptive?

Educational Technology researchers often oppose descriptive vs. prescriptive view of Instructional Design methods. The descriptive view is the objective study of the activity effectively carried out, the prescriptive one is the activity which should be. The purpose of this paper is to use the descriptive-prescriptive framework to present research in the Instructional Design field. We consider alternately behavioristic, media-centered, systemic, cognitive, as well as constructivist research. This framework allows us to cross descriptiveprescriptive approaches with origin and use of Instructional Design methods. We claim that these approaches are both necessary.

a planification d'une activité est une tâche familière accomplie régulièrement dans la vie quotidienne, par exemple lorsque nous nous apprêtons à cuisiner, effectuer un 
long voyage ou préparer le contenu de notre porte-documents. II est difficile d'affirmer que ces différentes activités peuvent se réaliser sans planification ou de préconiser une seule méthode pour s'en acquitter. Pourtant, ces avis sont souvent émis à propos de planification de l'enseignement. De nombreux ouvrages de formation d'enseignants proposent une méthode unique de conception de séquences d'enseignement, sans éclaircir leurs présupposés théoriques. On a pu également demander à des enseignants, sujets d'expérimentations, d'enseigner un contenu sans mettre en œuvre une planification préalable.

Notre avis, que nous allons détailler ici, est que tous les enseignants anticipent, de manière plus ou moins formelle, le contenu (qu'enseigner ?) et les activités (comment enseigner ce contenu ?) qu'ils vont proposer à leurs élèves. Planifier son enseignement est une activité quotidienne dont l'importance a été souvent mise en avant, bien que parfois de manière ambiguë : on est d'accord sur le principe selon lequel tous les enseignants planifient en partie leur activité et celle de leurs élèves. En revanche, leur façon de procéder, ainsi que les méthodes d'observation de cette activité, ne font pas l'objet de consensus. Cette manière d'observer la planification de l'enseignant a été qualifiée de descriptive, c'est la vue du chercheur. Une autre manière, qualifiée de prescriptive, se préoccupe de ce que l'enseignant devrait faire lorsqu'il planifie son enseignement, c'est la vue du formateur ou du supérieur hiérarchique. Pour résumer, les vues prescriptive et descriptive à propos de la conception de l'enseignement sont respectivement «Comment puis-je développer des procédures, des conventions, et des manières de considérer un enseignement comme bon ? » et «Comment en vient-on à apprendre [et à enseigner] ? » (Wilson, 1995)

Ces visions prescriptive et descriptive du processus d'enseignement paraissent diviser les chercheurs (voir des débats à ce sujet, notamment dans Bru, 1994, 1998 ; Crahay, 1998 ; de Ketele, 1986 ; Viau, 1993). Sans entrer tout de suite dans les controverses, signalons d'abord que la nature - l'origine - descriptive ou prescriptive d'un travail est souvent difficile à déterminer. Ainsi, pour donner un seul exemple, la taxonomie des objectifs de Bloom et al. (1969), un des travaux les plus cités dans le domaine éducatif, est-elle prescriptive (comme le pense Provencher, 1984) ou bien descriptive (comme le soutiennent de Landsheere et de Landsheere, 1984) ? Nous tenterons plus loin d'éclaircir ce point. 
Bruner (1970) et Simon (1974) ont pris part à ce débat et ont élaboré des distinctions entre description et prescription souvent reprises. Bruner a montré que le processus d'enseignement est essentiellement prescriptif, alors que le processus d'apprentissage est descriptif. «Une théorie de l'enseignement est prescriptive en ce qu'elle présente des règles à propos de la meilleure façon d'acquérir des savoirs ou savoir-faire. " (Bruner, 1970 , p. 112) Simon a distingué la science des phénomènes naturels, qui se préoccupe de définir la manière dont ces derniers sont constitués et fonctionnent, de la science des phénomènes artificiels, ou de l'ingénierie. Cette dernière, prescriptive, se préoccupe de la conception et la fabrication d'artefacts. Simon montre que « [...] pour l'essentiel, la complexité [du] comportement [humain] résulte de son environnement, de sa recherche de bonnes conceptions » (id., p. 103), et qu'il convient d'étudier l'humain au travers d'une science de la conception.

Cet article est l'occasion de montrer comment on a pu décrire et prescrire une activité importante de l'enseignant : la planification de séquences d'enseignement. Notre propos n'est pas de passer en revue ce qu'est cette activité, ni de proposer un répertoire de méthodes du domaine (1). En revanche, nous allons recenser les différents courants qui se sont intéressés à cette activité, que ce soit pour la décrire ou en prescrire une méthode. Avant cela, commençons par décrire le champ de travail qui les englobe : celui des techniques éducatives (Educational Technologies).

\section{LES TECHNIQUES EDUCATIVES : CONCEPTION ET IMPLEMENTATION DE L'ENSEIGNEMENT}

Le champ d'étude des techniques éducatives englobe deux courants de recherche principaux qui sont d'une part celui de la conception de l'enseignement (Instructional Design) et celui de son implémentation en situation (Instructional Development). De manière générale, ce champ a pour objet de relier les événements d'enseignement aux processus et résultats d'apprentissage. Le champ de travail de l'Instructional Design est né dans le domaine de l'entraînement ou de la formation d'employés dans l'industrie ou le commerce (Derry \& Lesgold, 1996). Le second, celui de l'implémentation, a pris naissance avec l'utilisation à des fins éducatives de moyens audiovisuels comme la radio, la télévision puis la micro-informatique.

L'étude des techniques éducatives s'est intensivement développée durant les années soixante. On peut remonter au moins jusqu'à l'entre-deux-guerres pour en trouver les 
premières traces (2). Pendant cette période, il a fallu former de très nombreux militaires au maniement d'armes ; à l'instar de nombreux progrès technologiques, la planification de l'enseignement a pris un tournant plus systématique avec son utilisation à des fins militaires. Que ce soit pour la formation accélérée à l'utilisation d'armes à feu (Wiburg, 1995) ou bien pour la mise au point d'entraînements au pilotage d'avions à réaction (Montague, 1986), il a fallu tenter d'optimiser, rationaliser la planification de séquences d'entraînement ou de formation. L'utilisation de plus en plus intensive de moyens audiovisuels (diapositives, radio, télévision, puis ordinateur) a mené au courant de recherche sur la technologie des médias, visant à réfléchir sur leur intégration dans l'enseignement. Le courant systémique, qui a suivi, a rompu avec une certaine vision parcellaire de la tâche, pour considérer l'ensemble du processus de l'enseignement/apprentissage. Ensuite des considérations plus microscopiques lui ont succédé, avec le courant cognitiviste. Actuellement, le courant de la cognition située, d'inspiration constructiviste, est l'objet de nombreux travaux, toutefois plus axés sur l'apprentissage que sur la planification.

Nous allons ici nous centrer sur l'Instructional Design et, plus précisément, sur ce qui a trait à la planification de séquences d'enseignement. Les raisons en sont les suivantes. Tout d'abord, c'est à notre avis l'activité de l'enseignant la moins étudiée et les définitions qui ont pu être énoncées sont souvent problématiques (3). Ensuite, on a pu considérer la phase de conception de l'enseignement comme une technique à part entière, donc fortement empreinte de prescription. Enfin, les multiples courants qui se sont intéressés à cette activité ont rendu peu lisibles les fondations théoriques de la planification : que doit son étude au behaviorisme, à l'étude de la technologie des médias, à la systémique, au cognitivisme ou au constructivisme?

Indiquons les principales caractéristiques de l'activité de planification de séquences d'enseignement. Les buts de la planification sont multiples : - apprendre, se remémorer le contenu à enseigner ; - préparer ou acquérir le matériel ou le curriculum ; - prendre des décisions sur le contenu, la durée, l'ordonnance et la clarté du matériel à enseigner.

En bref, planifier, c'est répondre à deux types de questions : «Sur quoi vais-je travailler », " Comment vais-je présenter cela au mieux à mes élèves ? » On a montré que la planification est un processus idiosyncrasique qui n'est pas orienté par la production d'une solution unique, mais un ensemble de solutions. Pour reprendre le terme de Bereiter 
(en préparation), on peut considérer que le produit de la planification est un artefact cognitif : - il peut être utilisé comme un outil, c'est-à-dire qu'il facilite le travail de l'enseignant ; - il sert à rationaliser le comportement de ce dernier.

Passons maintenant à l'exposé de notre cadre qui permettra de présenter quelques travaux des principaux courants ayant étudié la planification de séquences d'enseignement.

\section{LA PLANIFICATION DE SEQUENCES D'ENSEIGNEMENT, OBJET DE DESCRIPTION ET DE PRESCRIPTION}

Nous avons choisi de présenter l'évolution des travaux sur l'activité de planification de l'enseignement selon la manière dont ces derniers utilisent le couple description/prescription. On peut décomposer la grande majorité des études de cette activité de la manière suivante: les chercheurs se dotent d'un outil d'observation ou d'analyse de l'activité et, après l'observation effective, analysent des résultats, tirent des conclusions sur cette activité. À travers la présentation de travaux en ce domaine (voir Tableau II ci-après), on sera amené ici à mettre en relation l'origine de l'outil d'observation choisi avec les usages qui en ont été faits dans l'étude. L'origine correspond à l'intention des concepteurs de l'outil d'observation ; l'usage correspond à la manière dont des chercheurs se servent de cet outil. Cette relation est loin d'être toujours élucidée par les chercheurs, ce qui pose le problème de leur pertinence et de leur cohérence. Est-il pertinent d'utiliser une méthode prescriptive pour décrire une activité ? À l'inverse, que penser des travaux où l'on justifie des prescriptions en leur plaquant une grille d'observation descriptive ? En tous cas, il nous paraît insuffisant de considérer l'usage de méthodes sans se référer à leur origine théorique.

Ces deux modalités, origine et usage, nous permettent de proposer un cadre de présentation des travaux en matière de planification de séquences d'enseignement. Quatre démarches sont maintenant détaillées. Nous présentons en premier les deux activités descriptive et prescriptive (respectivement l'évaluation et l'analyse), puis les activités hybrides, mêlant description et prescription.

- La démarche d'évaluation fait un usage prescriptif de méthodes d'origine prescriptive. Elle vérifie que les prescriptions préalables - provenant par exemple d'une méthode de planification - sont effectivement mises en œuvre dans l'activité des sujets, sans 
qu'il soit porté un jugement de valeur sur cette mise en œuvre. C'est en partie le type d'activité que réalise un enseignant dans la phase postactive.

- La démarche d'analyse fait un usage descriptif de méthodes d'origine descriptive. Elle consiste à recueillir des traces de l'activité à partir d'un matériel descriptif et à décrire les résultats mis au jour. Cela revient à poser la question suivante : « Les descriptions de cette activité sont-elles fidèles à la réalité ? ", ainsi qu'à mettre à l'épreuve un modèle de l'activité. Cette démarche, contrairement à la précédente, n'a aucune intention prescriptive. La pertinence de son usage est ainsi soumis à la validation et peut être réfuté. C'est notamment l'activité que réalise un psychologue expérimentaliste, lorsqu'il observe, via une grille, les performances de sujets.

- la démarche de la rétro-ingénierie, ou l'ingénierie intuitive (reverse engineering) fait un usage descriptif de méthodes d'origine prescriptive. Elle revient à analyser, décomposer pas à pas l'activité afin de se demander comment elle est mise en œuvre dans une situation donnée. Cette démarche est pratiquée chaque fois qu'il est nécessaire de comprendre « comment ça marche » ou «comment c'est fait ». Elle est par exemple pratiquée dans des situations d'espionnage industriel - on ne connaît pas une technique, on l'analyse pour pouvoir la répliquer - ou dans la recherche en neuropsychologie - on ne connaît pas le fonctionnement du cerveau dans une activité précise, on reconstruit à rebours ce processus en partant de son activité obtenue par imagerie cérébrale (Pinker, 2000).

- La démarche de vérification fait un usage prescriptif de méthodes d'origine descriptive. Elle revient à se demander si les descriptions de l'activité sont bien celles qui ont été prévues avant la mise en œuvre de cette dernière, ou si elles sont bien compatibles avec les modèles existants. Elle diffère de la démarche d'évaluation (voir ci-dessus) en ce que l'on recherche ici les écarts à la prescription plutôt que la mise en œuvre de l'activité dans de bonnes conditions, même si des différences avec la prescription sont relevées. Elle est notamment appliquée dans des contextes de recherche en ergonomie.

Nous montrerons qu'il est préférable qu'une méthode soit utilisée de la même manière qu'elle a été initialement formulée. Ainsi, l'usage descriptif de principes d'origine descriptive et l'usage prescriptif de principes prescriptifs sont tout à fait pertinents et correspondent à des besoins que nous identifierons. En revanche, il faut considérer avec précaution les usages croisés : une méthode descriptive n'est parfois pas compatible avec des mises en œuvre prescriptives, et vice versa. Toutefois, il arrive que ces démarches 
croisées soient utiles : c'est notamment le cas en formation des enseignants, où les prescriptions des formateurs peuvent utilement s'appuyer sur les données de la recherche. Le tableau I ci-dessous reprend ces quatre démarches.

Tableau I - Vue générale des démarches selon le couple description/prescription

\begin{tabular}{|c|c|c|}
\hline & Usage prescriptif & Usage descriptif \\
\hline $\begin{array}{l}\text { Origine prescriptive } \\
\text { La méthode de planification } \\
\text { prescrit une démarche }\end{array}$ & $\begin{array}{l}\text { Démarche d'évaluation } \\
\text { Cette méthode est-elle correctement } \\
\text { appliquée par les enseignants? }\end{array}$ & $\begin{array}{l}\text { Démarche de la rétro-ingénierie } \\
\text { Cette méthode modifie-t-elle } \\
\text { réellement - et en quoi ? - } \\
\text { l'enseignement ou l'apprentissage? }\end{array}$ \\
\hline $\begin{array}{l}\text { Origine descriptive } \\
\text { On observe des enseignants } \\
\text { au travail }\end{array}$ & $\begin{array}{l}\text { Démarche de vérification } \\
\text { Cette activité est-elle compatible avec } \\
\text { la méthode de planification? } \\
\text { Les enseignants se comportent-ils en } \\
\text { classe comme ils l'ont prévu? } \\
\text { Le travail des enseignants est-il effi- } \\
\text { cace? }\end{array}$ & $\begin{array}{l}\text { Démarche d'analyse } \\
\text { Les descriptions de cette activité sont- } \\
\text { elle fidèles à la réalité ? }\end{array}$ \\
\hline
\end{tabular}

Examinons maintenant, à partir de ce cadre, l'évolution des travaux sur la planification de séquences d'enseignement.

\section{LE COURANT BEHAVIORISTE, OU L'ENSEIGNANT COMME SELECTIONNEUR DE TACHES}

Ce courant, inspiré des idées de Skinner, voit l'enseignement comme résultant d'une décomposition amenant à la sélection de certaines tâches. Il a pris son essor au sein de l'enseignement programmé, qui peut être vu comme une mise en œuvre algorithmique de conception de séquences d'enseignement : le contenu est présenté pas à pas, les feedback sont nombreux et immédiats, ce qui simule une adaptation à l'élève. Développons, à partir de notre cadre, les principaux travaux de ce domaine.

Démarche d'évaluation. Tyler, il y a cinquante ans, a établi quatre «questions fondamentales auxquelles répondre pour développer n'importe quel curriculum ou cours » (Kliebard, 1995, p. 81, citant Tyler, 1950) : - Quels buts éducatifs sont poursuivis ? Quelles expériences d'apprentissage sont proposées aux élèves afin que ces buts soient atteints ? - Comment ces dernières sont-elles organisées ? - Comment déterminer si les buts ont été atteints ? Cette démarche de découpage de tâches a également été mise en œuvre dans l'enseignement programmé (Skinner, 1968), à la fin des années cinquante. Mager (1977) a réalisé dans cette même veine une méthode de définition d'objectifs péda- 
gogiques, indépendants du contenu, que Glaser étend à l'évaluation (tests critériés). De ce découpage des tâches et des conditions de l'apprentissage mis au jour par Tyler, puis Gagné, est issue la grande majorité des méthodes de planification de séquences d'enseignement, qui ont concouru à faire spécifier par les enseignants des objectifs «bien formés ».

Démarche d'analyse. Les taxonomies d'objectifs posent qu'il existe une hiérarchie de performances d'apprentissage (de la plus simple ou facile, à la plus complexe ou difficile) et, pour chaque performance, qu'il existe une condition y menant. II est également préférable de formuler ces performances en termes d'actions, hiérarchisées. Ces taxonomies, notamment celle de Bloom et al. (1969), ont été conçues pour décrire la formulation des divers documents institutionnels sur l'éducation. Elles ont donc clairement une origine descriptive, origine qui a curieusement été occultée, comme nous l'avons signalé dans l'introduction. Paradoxalement, l'utilisation descriptive de telles taxonomies n'est pas courante. Toutefois, des travaux menés par Kibler et al. (1974, cités par Burton, Moore \& Magliaro, 1996) ont montré que la spécification des objectifs d'enseignement et leur divulgation auprès des élèves s'accompagnaient rarement d'un meilleur apprentissage de ces derniers. Ces résultats en préfigurent d'autres, ultérieurs, montrant qu'une telle démarche conclut souvent à une différence non significative entre le groupe expérimental et le groupe-contrôle (voir ci-dessous et Lockee, Burton \& Cross, 1999).

Démarche de la rétro-ingénierie. En revanche, on a beaucoup utilisé les taxonomies comportementales évoquées ci-dessus à des fins prescriptives. Cela a mené à un mélange des genres peu cohérent, comme l'indiquent de Landsheere et de Landsheere (1984, p. 67): «La façon d'utiliser les taxonomies n'est pas toujours bien comprise. Souvent, on a voulu en faire un instrument destiné à formuler des objectifs, alors qu'il est d'abord destiné à analyser des objectifs existants ". On a là un exemple-type de décalage entre origine et usage : les taxonomies, initialement descriptives (4) peuvent difficilement remplir un rôle prescriptif.

Démarche de vérification. L'approche prescriptive par l'analyse descriptive de la tâche, telle qu'elle a été enseignée dans le cadre de la pédagogie par les objectifs, a fait que les enseignants ont été parfois obligés de modifier leurs travail pour le faire cadrer avec la méthode préconisée. On a d'ailleurs souvent ironisé sur la formule "L'élève doit être capable de... » qui transformerait de facto une visée d'enseignement en objectif bien 
formé. Un autre ensemble de travaux a vu le jour sous la dénomination de « recherches processus-produit » (Dunkin \& Biddle, 1974), menées dans les années soixante jusqu'au début des années soixante-dix. Ces travaux, s'ils n'ont pas directement concerné l'activité de planification, ont pu déterminer des comportements efficaces d'enseignants à partir de séries d'observations in vivo et des mesures de performance d'élèves. On a là, typiquement, des études d'origine descriptive (observer les comportements des enseignants et des élèves), donnant lieu à des considérations prescriptives (on en dégage des critères d'efficacité). II a beaucoup été écrit à propos des avantages et inconvénients de ces recherches (Shulman, 1986). Le principal écueil est que ces recherches ont voulu justifier l'efficacité de l'enseignant à partir de méthodes d'origine descriptive non conçues à cet effet (5).

Tableau II - Position des travaux behavioristes par rapport à la description et la prescription

\begin{tabular}{lll}
\hline & Usage prescriptif & Usage descriptif \\
\hline $\begin{array}{l}\text { Origine } \\
\text { prescriptive }\end{array}$ & $\begin{array}{l}\text { Démarche d'évaluation } \\
\text { Méthodes de planification (Tyler, 1950) } \\
\text { Enseignement programmé (Skinner, 1968) }\end{array}$ & \\
$\begin{array}{l}\text { Origine } \\
\text { descriptive }\end{array}$ & $\begin{array}{l}\text { Démarche de vérification } \\
\text { Pédagogie par les objectifs (Mager, 1962) } \\
\text { Recherches processus-produit (Dunkin \& } \\
\text { Biddle, 1974) }\end{array}$ & $\begin{array}{l}\text { Démarche d'analyse } \\
\text { Taxonomie des objectifs (Bloom et al., }\end{array}$ \\
\hline
\end{tabular}

La principale difficulté de ce courant behavioriste est que l'on a été - et l'on est d'ailleurs encore - dans l'impossibilité de définir de manière ordonnée des tâches aussi "simples » que celles d'écrire ou de lire (Rieben, 1988), impossibilité qui rend de fait encore plus difficile la description de tâches plus complexes. Toutefois, ce courant a eu une descendance importante : à partir de lui, on a considéré la planification comme un processus séquentiel, systématique et dirigé par les buts. Les chercheurs vont ensuite se recentrer sur les techniques et se préoccuper davantage de la manière dont les élèves reçoivent les informations.

\section{LE COURANT DE LA TECHNOLOGIE DES MEDIAS, OU L'ENSEIGNANT COMME SELECTIONNEUR DE MEDIAS}

Si les recherches en enseignement programmé ont bénéficié des principes théoriques solides - bien que controversés - de Skinner, le courant centré sur les médias qui a suivi (voir Tableau III ci-après) a eu des difficultés à asseoir des principes du même ordre. 
Des chercheurs en enseignement programmé, dont Montmollin (1971), ont été réticents à considérer les techniques audiovisuelles comme à même de favoriser l'apprentissage : «[...] les méthodes de l'enseignement programmé ont plus apporté aux aides audiovisuelles que celles-ci n'ont apporté à la programmation » (id., p. 32). Deux principes ont toutefois été attachés à ce courant (Eraut, 1996) : - la richesse et la diversité des stimuli offerts à l'apprenant sont censés améliorer sa motivation et son attention ; - les médias permettent un degré d'abstraction croissant dans la présentation des systèmes de symboles (principe notamment utilisé par Dale, 1969, cité par Levie, 1989, avec une représentation unidimensionnelle du degré d'abstraction des médias).

Démarche d'évaluation. La popularisation du matériel audio-visuel a permis de développer les premiers cours « multi-média » (de Corte et al., 1979). C'est à ce moment que l'on esquisse des méthodes de conception de séquences d'enseignement qui tiennent compte de la manière dont les élèves apprennent, afin de transférer l'éventuelle efficacité des enseignants sur les médias. La question, à l'origine, est purement prescriptive : «Soit un problème d'enseignement spécifié par un ensemble d'objectifs, un groupe d'apprenants et une situation d'enseignement, comment l'enseignant sélectionne le média ou la combinaison de médias par laquelle il délivrera son enseignement ? » (Levie, 1989, p. 398). Elle est aussi devenue plus descriptive quand il s'est agi de mesurer d'éventuels effets de médias sur l'apprentissage et la compréhension.

Démarche d'analyse. Le débat sur la description de l'effet des médias sur l'apprentissage a donné lieu à de nombreux échanges de points de vue à partir de deux idées principales (Dessus \& Lemaire, 1999) :

- Le média n'influence jamais l'apprentissage, car il est seulement le véhicule qui délivre l'enseignement, il n'incorpore pas la méthode d'enseignement. Clark (1983) argumente cette idée en citant les très nombreuses études expérimentales intermédias concluant à des différences non significatives.

- Le média influence l'apprentissage et, si peu de travaux parviennent à mettre au jour une différence intermédia significative, c'est d'une part parce qu'une adaptation aux fonctionnalités de chaque média est nécessaire ; d'autre part, parce que la métaphore du véhicule de l'information est inadéquate pour décrire l'apprentissage de connaissances nouvelles lors de l'apprentissage. En effet, les courants constructivistes 
et surtout celui de la cognition située remettent en cause une telle conception (voir plus loin, ainsi que Kozma, 1991).

Ce débat a une incidence sur le travail de planification de l'enseignant : si l'on pense que le média est un simple véhicule qui achemine l'information, on va porter son intérêt sur les méthodes d'enseignement qui utilisent, de manière optimale un certain média ; si l'on pense que le média influe sur l'apprentissage, alors on tentera de concevoir ou d'utiliser des médias qui incorporent à la fois des méthodes d'enseignement efficaces, et des moyens de construire de nouvelles connaissances.

Démarche de vérification. Faute de théorie pertinente sur l'apprentissage par les médias, l'intérêt s'est porté sur une vision plus globale de l'enseignement. Le travail descriptif nommé «paradigme des processus médiateurs " (mediating processes), qui s'inscrit dans ce champ, a pris pour objet d'étude les activités cognitives des élèves à travers les réponses qu'ils livrent à l'enseignant. Cette centration sur l'élève atténue le rôle de l'enseignant, mais a été le véritable précurseur d'études ultérieures sur les processus cognitifs. Elle rompt en cela avec le courant behavioriste, qui considère l'apprentissage et l'enseignement comme observables, externes à l'élève et à l'enseignant. Toutefois, un des problèmes attachés à ce paradigme - outre le fait qu'il a peu à voir avec les médias d'enseignement en tant que tels - est qu'il a été majoritairement utilisé à des fins prescriptives. Doyle (1986), en faisant une revue des applications de ce paradigme, montre qu'il a été rapidement associé à des prescriptions pour enseignants : présenter clairement un cours, accorder de l'importance aux gestes et mouvements, évaluer l'attention des élèves. Ces prescriptions, pour utiles qu'elles soient, ont détourné les chercheurs de l'étude des processus cognitifs des élèves.

Démarche de la rétro-ingénierie. La grande majorité des méthodes de planification de séquences d'enseignement, notamment celles faisant intervenir différents médias, sont restées à un tel niveau de généralité qu'elles ne varient pas selon le contenu ou le média utilisé (Sauvé, 1993 ; Trollip, 1996, proposent diverses méthodes pour la conception de cours en tenant très peu compte du média). D'origine prescriptive, et a priori conçues quel que soit le média utilisé, ces méthodes de planification ne peuvent, a posteriori, en tenir compte. Tout se passe comme si l'on avait redécomposé des méthodes de planification pour y intégrer des médias, seulement du fait qu'ils sont disponibles. 
Tableau III - Position des recherches sur la technologie des médias par rapport à la description et la prescription

\begin{tabular}{lll}
\hline & Usage prescriptif & Usage descriptif \\
\hline $\begin{array}{l}\text { Origine } \\
\text { prescriptive }\end{array}$ & $\begin{array}{l}\text { Démarche d'évaluation } \\
\text { Le multi-média (Dale, 1969) }\end{array}$ & $\begin{array}{l}\text { Démarche de la rétro-ingénierie } \\
\text { Prise en compte des médias dans la } \\
\text { planification (Sauvé, 1993; Trollip, 1996) }\end{array}$ \\
$\begin{array}{l}\text { Origine } \\
\text { descriptive }\end{array}$ & $\begin{array}{l}\text { Démarche de vérification } \\
\text { Les processus médiateurs (Doyle, 1986) }\end{array}$ & $\begin{array}{l}\text { Démarche d'analyse } \\
\text { Effet des médias (Clark, 1983; Kozma, 1991) }\end{array}$ \\
\hline
\end{tabular}

Ces différentes recherches sur les médias ont été répliquées dans les courants suivants, du moins par leurs problématiques : mesurer d'éventuels effets de médias ou de concevoir des grilles de conception d'enseignements adaptées à un média. Aborder l'enseignement par la technique est donc, on le constate encore de nos jours, une piste souvent empruntée.

\section{LE COURANT SYSTEMIQUE OU L'ENSEIGNANT COMME GESTIONNAIRE DE VARIABLES}

Le courant systémique entretient une parenté avec l'enseignement programmé, via la cybernétique : importance de la communication, des feed-back, du contrôle de l'enseignant, qu'il soit direct ou par le biais d'une machine. Sa problématique principale est de rendre compte de phénomènes complexes, non contrôlables et non déterministes. Le courant systémique a donc consisté, en ce qui concerne la planification de séquences d'enseignement, en la formulation de nombreux «modèles », prenant en compte des aspects macroscopiques du fait éducatif (voir Tableau IV ci-après).

Démarche d'évaluation. Dans les années soixante-dix, des travaux ont procédé en l'inventaire d'un très grand nombre de variables visant à appréhender des situations éducatives, à des fins de conception (Berbaum, 1982) ou de régulation (d'Hainaut, 1980). Après une période de relatif désintérêt, ces méthodes systémiques ont bénéficié d'un regain d'attention de la part de réformateurs de systèmes éducatifs, à travers la notion de «pilotage » (de Landsheere, 1994 ; Romiszowski, 1996). Toutefois, l'incidence dans les établissements de ce «pilotage » de systèmes éducatifs ne s'est peut-être pas encore fait sentir. Selon Dick (1992, cité par Derry \& Lesgold, 1996, p. 788) : «On ne peut attribuer aux instructional designers les mauvaises performances des élèves, car ils n'ont pratiquement pas contribué à l'élaboration du programme d'enseignement des écoles publiques américaines » 
Démarche d'analyse. Les méthodes de planification des courants précédents pouvaient intégrer des phases évaluatives, mais ces dernières étaient peu précises. Les techniques évaluatives se popularisant et se raffinant, la centration sur ces dernières ne tarde pas et l'on prend position parmi les techniques offertes : évaluation pronostique, sommative, formative, critériée, etc. On voit donc apparaître des méthodes avec une phase d'évaluation pour chacune des phases de la conception (6) ; ainsi qu'un type d'évaluation pour chaque phase de l'enseignement (pronostique pour le préactif, formative pour l'interactif, sommative pour le postactif). Les méthodes de planification du courant systémique sont ainsi largement orientées vers l'évaluation.

Démarche de la rétro-ingénierie. Les productions d'inspiration systémique ont souvent été sujettes à critique, parfois injustement :

« II y eut une époque où l'on produisait à la tonne des modèles systémiques de planification ! Ils furent, pour la plupart, admirés sans être utilisés. On louangeait l'auteur, on admirait ses schémas à multiples boîtes et aux nombreuses flèches qui se renvoyaient les unes aux autres, et on rangeait, par la suite, son livre à la bibliothèque ! »(Bertrand, 1993, p. 10)

Le regret de Bertrand semble être que ces «modèles " prescriptifs (qui sont sans doute des méthodes) n'ont pas donné lieu à des réalisations concrètes. Mais était-ce vraiment leur rôle ? Ces «modèles » prescriptifs doivent-ils être réalistes, c'est-à-dire utilisés à des fins descriptives ? (7) Si c'est le cas, c'est adopter une démarche de rétroingénierie. On peut même se demander si ces «modèles" ne sont pas en fin de compte utilisés, que ce soit à titre prescriptif (ne sont-ils pas utilisés en formation ?) ou descriptif (ne décrivent-ils pas certaines pratiques ?). 
Tableau IV - Position des recherches systémiques par rapport à la description et la prescription

\begin{tabular}{lll}
\hline & Usage prescriptif & Usage descriptif \\
\hline $\begin{array}{l}\text { Origine } \\
\text { prescriptive }\end{array}$ & $\begin{array}{l}\text { Démarche d'évaluation } \\
\text { Pilotage de systèmes éducatifs (de } \\
\text { Landsheere, 1994) }\end{array}$ & $\begin{array}{l}\text { Démarche de la rétro-ingénierie } \\
\text { Utilité des « modèles »? (Bertrand, 1993) }\end{array}$ \\
$\begin{array}{l}\text { Origine } \\
\text { descriptive }\end{array}$ & - & $\begin{array}{l}\text { Démarche d'analyse } \\
\text { Méthode de planification intégrant des techniques }\end{array}$ \\
& & $\begin{array}{l}\text { évaluatives (Dick \& Carey, 1990; Tennyson, } \\
1993)\end{array}$ \\
\hline
\end{tabular}

Le courant systémique a donné lieu, nous l'avons vu, à des travaux très divers. Ces derniers ont été réutilisés dans le courant cognitiviste qui suit, comme les nombreuses méthodes de planification intégrant les nouvelles techniques évaluatives.

\section{LE COURANT COGNITIVISTE, OU L'ENSEIGNANT COMME PSYCHOLOGUE}

L'apport de la psychologie cognitive à l'éducation s'est fait assez tardivement (Bonner, 1988). Ou plutôt, comme l'indique Wilson (1995), les spécialistes de la conception de séquences d'enseignement n'ont pas suivi les préoccupations des psychologues, qui ont abordé les dimensions cognitives de l'apprentissage dès les années soixante-dix. C'est donc avec retard que, dans le champ de la planification, on s'est intéressé aux aspects cognitifs de l'activité d'enseignement. Pour les chercheurs de ce courant (voir Tableau V ci-après), l'enseignant n'est plus considéré comme un analyseur de tâche, un sélectionneur de média ou un gestionnaire de variables ; il doit, en fonction des connaissances des élèves, formuler des situations d'apprentissage variées, dans lesquelles l'élève acquerra progressivement le contenu à apprendre.

Démarche d'évaluation. A. C. Young, Reiser et Dick (1998) ont réalisé une étude dans laquelle des enseignants experts étaient interrogés sur leur manière de réaliser diverses planifications, selon des échéances différentes. Les résultats montrent que les sujets ne suivent pas les procédures habituellement prescrites : ils ne spécifient notamment pas d'objectifs, ils ne se réfèrent pas à l'évaluation des élèves. On remarque, dans la littérature, des positions très divergentes à propos de ce type de résultat : faut-il enseigner ou non des procédures qui ne sont pas celles observées chez des enseignants plus expérimentés? Les auteurs de l'étude précédente adoptent une position médiane : pour eux, il est utile de signaler aux enseignants en formation initiale que leurs collègues plus 
expérimentés ne planifient pas selon les méthodes en vigueur. II importe pour autant de les leur enseigner : cela les expose à un grand nombre de variables éducatives, qu'ils auront par la suite la possibilité de filtrer et d'éliminer, afin de se forger leur propre méthode, correspondant à la fois à leurs caractéristiques personnelles et au contexte dans lequel ils travailleront.

Démarche d'analyse. Une des études les plus citées sur la cognition de l'enseignant à origine et usage descriptifs est sans doute celle de Leinhardt et Greeno (1986). Ces auteurs mettent au jour, par une observation d'une douzaine d'enseignants experts et novices, une dizaine de scripts d'enseignement, répertoire de routines que ces derniers utilisent, notamment pour réduire leur charge cognitive et rendre leur action prédictible par leurs élèves. Dans un travail étudiant l'accès des enseignants aux connaissances de la situation, Bru (1991) (8) a montré que l'activité de l'enseignant pouvait être décrite en une douzaine de variables d'action, qu'il classe en trois catégories. Les première et troisième catégorie concernent plutôt la phase préactive de l'enseignement, alors que la deuxième regroupe des variables processuelles, donc modifiées durant la phase interactive. Des planifications ont été analysées selon ces variables et Bru a montré que, lorsqu'on donne comme consigne aux enseignants de réaliser des planifications « [les] plus contrastée[s] possible» (id., p. 125), la variabilité des planifications des enseignants est importante : près du tiers d'entre eux font varier plus de huit variables d'action, mais cette variabilité se trouve moins marquée lorsque les enseignants enseignent selon ce qu'ils ont planifié.

Démarche de la rétro-ingénierie. Cette démarche a été souvent utilisée dans ce courant, où il s'est agi de valider de manière descriptive des méthodes prescriptives de planification de séquences d'enseignement : « quel est l'effet réel de ces prescriptions sur les enseignants, lorsqu'ils les suivent ? » et non pas seulement : « appliquent-ils bien les prescriptions qu'on leur enseigne ? » (Klein, 1991) Cette question a donné lieu, dans la lignée de Zahorik (1975), à de nombreux travaux (Altet, 1994 ; Kerr, 1981 ; Klein, 1991). Par exemple, Altet (1994) a réalisé une analyse descriptive générale du processus d'enseignement/apprentissage, à partir d'observations de l'activité d'une centaine d'enseignants et de questionnaires. Altet demande à des enseignants de réaliser une planification, structurée en rubriques, qu'elle analyse par la suite. L'activité est ainsi prescrite dans une certaine mesure, même si certains enseignants ne suivent pas ces prescriptions et réalisent une planification libre (9). 
Tableau V - Position des recherches cognitivistes par rapport à la description et la prescription

\begin{tabular}{lll}
\hline & Usage prescriptif & Usage descriptif \\
\hline $\begin{array}{l}\text { Origine } \\
\text { prescriptive }\end{array}$ & $\begin{array}{l}\text { Démarche d'évaluation } \\
\text { Les enseignants efficaces } \\
\text { appliquent-ils les méthodes } \\
\text { prescrites ? (Young et al., 1998) }\end{array}$ & $\begin{array}{l}\text { Démarche de la rétro-ingénierie } \\
\text { Les enseignants appliquent-ils les méthodes } \\
\text { prescrites ? (Kerr, 1981; Klein, } 1991 ; \text { Zahorik, 1975) }\end{array}$ \\
$\begin{array}{l}\text { Origine } \\
\text { descriptive }\end{array}$ & - & $\begin{array}{l}\text { Démarche d'analyse } \\
\text { Routines d'enseignement (Leinhardt \& Greeno, 1986) } \\
\text { Variabilité des conduites enseignantes (Bru, 1991) }\end{array}$ \\
\hline
\end{tabular}

II y a deux écueils principaux attachés à ce courant. Tout d'abord celui du statut des verbalisations des enseignants, que Yinger (1986) a examiné de manière méthodique, en montrant les problèmes de validité des méthodes de rappel stimulé (10). Ensuite, le fait que ces méthodes ne soient pas fondées sur une théorie de l'apprentissage : l'enseignant est considéré hors de toute référence à ses élèves et à la manière dont ils apprennent.

\section{LE COURANT CONSTRUCTIVISTE ET LA COGNITION SITUEE, OU L'ENSEIGNANT COMME TUTEUR}

La notion de planification de l'enseignement selon une approche constructiviste est un oxymoron, tant l'enseignement n'est plus considéré comme une démarche où l'enseignant programme seul son activité future. Le rôle de l'élève est reconsidéré, ce dernier devient lui-même un concepteur. Par rapport aux courants étudiés précédemment, le statut de la planification change : les plans ne précèdent plus les actions dans une perspective procédurale, fixée par avance, mais sont plutôt interprétés avec souplesse, selon le contexte, de manière non prédéterminée.

Le courant de la cognition située contient, contrairement à la plupart des précédents, des conseils explicites concernant la planification de séquences d'enseignement. Cela n'a paradoxalement pas entraîné beaucoup de travaux visant à préciser ces conseils (voir Tableau VI ci-après). La raison en est sans doute que les vues descriptives ne produisent, en tant que telles, que peu ou pas de principes prescriptifs. De plus, ce qui rend les prescriptions à propos de la planification difficiles à appliquer est le principe que la construction des connaissances se réalise sur-le-champ et non plus par une seule activité de mémorisation, comme avec le courant cognitiviste. Enfin, ce primat de l'environnement oblige les enseignants à tenir compte des contraintes de la situation, plutôt que d'essayer d'appliquer la meilleure méthode disponible. Les actions de l'enseignant ne peuvent être 
prédéterminées, puisqu'elles doivent être les plus spécifiques possible aux événements qui vont se produire en situation.

Démarche d'évaluation. M. F. Young (1993) indique quatre principes d'aide à la conception d'activités authentiques, dans lesquelles l'enseignant est vu comme un entraîneur. Ces principes, à origine et usage prescriptifs, sont des guides permettant de bâtir des séquences d'enseignement obéissant à des principes constructivistes. On retrouvera, en filigrane, certains items de la méthode de Tyler.

- Sélectionner la situation, ou l'ensemble des situations qui permettra l'acquisition des connaissances ;

- Procurer l'étayage (scaffolding) qui permettra aux novices (mais aussi aux experts) de travailler dans ce contexte ;

- Procurer des supports permettant à l'enseignant de garder trace des progrès des élèves, d'évaluer leurs productions, tout en permettant aux élèves d'interagir, de partager leurs connaissances, etc. ;

- Définir le rôle, la nature et la signification de l'évaluation.

Démarche d'analyse. Ce courant, à l'instar des précédents, n'a pas manqué de travaux testant sa validité. Gruber, Law, Mandl et Renkl (1995), en faisant une revue de la question des principales applications de l'apprentissage situé, montrent que le fait d'inclure, dans un logiciel, quelques caractéristiques réalistes ne suffit pas à le rendre efficace. Plus important encore, ces auteurs soulignent la difficulté de bâtir des tests évaluant le transfert d'habiletés acquises dans des environnements situés. En effet, de tels environnements promeuvent une activité de résolution de problèmes dans des domaines complexes et faiblement structurés, ce qui rend difficile l'évaluation d'éventuels transferts d'habiletés.

Démarche de vérification. L'idée centrale du courant constructiviste est que l'élève dispose de connaissances initiales du monde, à partir desquelles il en construit de nouvelles. Partant de cette idée, les chercheurs en techniques éducatives ont tenté de bâtir des environnements d'apprentissage « authentiques » (informatisés ou non), c'est-àdire correspondant au monde réel. Mais, comme l'indique Petraglia (1998), cette nouvelle contrainte est difficile à respecter et elle a amené les concepteurs à rendre ces situations authentiques a priori (l'auteur nomme cela la pré-authentification). Cela, en contradiction avec les idées constructivistes initiales. C'est en effet à l'élève d'être persuadé de 
l'authenticité du problème proposé. Le monde réel n'étant pas compris indépendamment des actions du sujet et donc ce sentiment d'authenticité ne peut être prédéterminé par quiconque (11).

\begin{tabular}{|c|c|c|}
\hline & Usage prescriptif & Usage descriptif \\
\hline Origine prescriptive & $\begin{array}{l}\text { Démarche d'évaluation } \\
\text { Principes de conception d'une } \\
\text { situation authentique (M. F. Young, } \\
\text { 1993) }\end{array}$ & - \\
\hline Origine descriptive & $\begin{array}{l}\text { Démarche de vérification } \\
\text { Pré-authentification (Petraglia, 1998) }\end{array}$ & $\begin{array}{l}\text { Démarche d'analyse } \\
\text { Études expérimentales (Gruber et al., } \\
\text { 1995) }\end{array}$ \\
\hline
\end{tabular}

Nous disposons actuellement de peu de recul pour rendre compte des avantages et inconvénients de ce courant. Nous pouvons seulement citer quelques débats à son propos. Certains chercheurs, comme Duffy et Cunningham (1996) ou Petraglia (1998) notent une continuité forte entre le courant constructiviste et celui de la cognition située. D'autres relèvent de nombreuses différences (Anderson, Reder \& Simon, 1995). On peut toutefois en mentionner la principale : le courant constructiviste pose que la connaissance réside dans l'individu, alors que le courant situé part du principe qu'elle est appréhendée de manière externe, sociale. Enfin, d'autres critiques sont venues de théoriciens de la conception de séquences d'enseignement comme Merrill et al. (1996). En se fondant sur les principes behavioristes et positivistes, ils montrent que le courant constructiviste ne conviendrait pas pour définir précisément ce que l'enseignant met en œuvre pour enseigner et ce que l'élève doit apprendre : en effet, le comportement et les performances des élèves ne peuvent être prédits que dans un contexte théorique où la connaissance est quantifiable, ce qui n'est pas le cas du courant constructiviste. Pourtant, selon ces auteurs, cette prédictibilité des performances est l'une des conditions essentielles de l'apprentissage. 


\section{DISCUSSION \\ PRESCRIRE ET DECRIRE EN PLANIFICATION DE SEQUENCES D'ENSEIGNEMENT}

Nous avons montré précédemment comment on a étudié ou prescrit le travail de planification de l'enseignant selon divers courants. On a souvent mis au jour des différences entre décrit et prescrit, comme autant de différences entre la réalité et le prévu. Signaler l'existence de ces différences n'est pas suffisant, il faut déterminer pourquoi elles existent. Cette détermination a été réalisée dans chaque courant, avec des résultats variables. II est intéressant de noter que les différences décrit/prescrit ont été diversement interprétées selon le courant de référence du chercheur. Le chercheur béhavioriste, face à une telle différence, invoquait une mauvaise application des taxonomies. Le chercheur en technologie des médias pouvait prétexter d'une mauvaise adéquation entre les caractéristiques du média et la situation. Le chercheur systémiste mettait en avant la nécessaire adaptation aux exigences du métier. Le cogniticien, en ergonome, invoque l'adaptation à la situation de travail. En ce qui concerne le courant de la cognition située, on ne semble pas, à notre connaissance, se préoccuper de justifier de telles différences. Discutons ici la place que l'on peut accorder aux vues descriptive et prescriptive à l'étude de ce travail.

\section{Réhabiliter la prescription}

La planification de séquences d'enseignement, en tant que technique, est fortement teintée de prescription. II nous paraît utile tout d'abord de réhabiliter la prescription en éducation. Cette démarche est trop souvent dévolue à des tâches d'inspection ou de formation alors qu'elle présente aussi un intérêt dans l'étude de l'activité de l'enseignant. Par exemple, en ergonomie, il est fait un usage précis de la comparaison entre tâche prescrite et effective (décrite) suivie par un opérateur. Cette comparaison permet notamment de mettre au jour le nécessaire processus d'adaptation de l'opérateur à la situation de travail.

Dans la recherche en éducation, les méthodes prescriptives et les observations descriptives à propos de l'enseignement sont élaborées avec des problématiques qui les relient rarement de manière fructueuse, c'est-à-dire, selon notre terminologie, par les démarches de vérification ou d'analyse. Les méthodes prescriptives ne sont en général pas testées en situation et les observations descriptives invalident les précédentes. Pour 
autant, ces méthodes prescriptives sont-elles inutiles ? Nous pensons qu'il existe des méthodes prescriptives cadrant suffisamment avec les situations réelles d'enseignement, et d'autres méthodes inadéquates, car trop éloignées de ces situations.

\section{Combiner prescription et description}

Toutefois, la réhabilitation de la prescription ne doit pas s'accompagner de l'abandon de la description. Carlgren (1999) a bien montré le danger d'étudier le travail de l'enseignant seulement sous l'angle descriptif. Cela revient, en particulier, à considérer les connaissances tacites, intuitives, de l'enseignant comme fausses ou à les remettre en cause, seulement parce qu'elles sont difficilement accessibles :

«On attend que les enseignants fassent et connaissent autre chose que ce qu'ils savent ou connaissent réellement. On ne met pas en doute la parole d'un médecin affirmant qu'il ne peut soigner un certain type de cancer. On considérera qu'un enseignant qui prétend qu'il ne peut enseigner un certain contenu à certains enfants a besoin d'être mieux formé. » (Carlgren, 1999, p. 48, c'est l'auteur qui souligne)

Une manière d'étudier la planification de l'enseignant qui concilierait description et prescription serait celle de Merrill et al. (1996), qui suit ces trois points :

- II y a différents types de connaissances et d'habiletés, à propos desquels on connaît différentes hiérarchies les décrivant (comme celle de Gagné) ;

- II y a différentes stratégies d'enseignement qui ont pu être élaborées et communiquées (décrites) par les enseignants et les chercheurs ;

- Pour chaque type de connaissance et d'habileté, on peut prescrire une stratégie d'enseignement qui serait appropriée.

\section{La double illusion théorie/pratique}

Jusqu'ici, nous avons volontairement évité l'usage du mot « théorie ». C'est curieusement un mot associé à la fois à la description et la prescription. Crahay (1998) signale que la démarche descriptive part de la description de faits éducatifs afin d'en dégager des principes théoriques (démarche nomothétique); Bru (1998) montre que la démarche prescriptive, de par son éloignement de la pratique, en vient à théoriser souvent abusivement les faits éducatifs. Les chercheurs débattant des vues prescriptives et descriptives 
en éducation en sont souvent venus à un débat associant prescription à « théorie fondée sur la pratique » (ou infondée, pour les détracteurs de la prescription) et description à «pratique théorisée » (ou insuffisamment théorisée pour les détracteurs de la description).

II nous paraît normal que ce débat interfère avec le débat plus large des différences théorie vs pratique, mais il nous paraît toutefois distinct. Carr (1990, p. 100) écrit à ce sujet : « II est doublement illusoire de penser que l'on "théorise" à propos d'éducation sans se demander comment cette éducation est "pratiquée", pendant qu'on "pratique" en éducation sans se demander comme cette pratique est théorisée ou comprise ». Carr signale cette illusion car il n'existe pas deux types d'études séparés qui seraient «comment théoriser en éducation » et «comment pratiquer». Ce qui pose souvent un problème pour les chercheurs, c'est la proximité des vues descriptives et prescriptives. Elles doivent être développées de manière parallèle, sans interférence l'une avec l'autre. La première permet de mieux connaître l'activité de l'enseignant, la seconde de mieux former ou superviser ce dernier. Nous avons montré que les théories d'origine prescriptive sont difficilement remises en cause par des usages descriptifs : même si l'on peut les invalider, elles n'en disparaissent pas pour autant de la circulation. De plus, il arrive qu'elles alimentent des travaux descriptifs. Les théories d'origine descriptive, elles, perdent souvent beaucoup à être validées de manière prescriptive et vice versa. Concluons par deux paraboles, exposant les démarches de la rétro-ingénierie et de la vérification dans le domaine plus large de la recherche en éducation.

\section{CONCLUSION}

\section{PARABOLES DE LA RETRO-INGENIERIE ET DE LA VERIFICATION}

La parabole de Papert (1981) illustre parfaitement l'intérêt de considérer comme également importantes les vues descriptives et prescriptives et montre de plus la difficulté de la démarche de la rétro-ingénierie, qui correspond à démonter, analyser, un produit technique, bâti à partir de principes prescriptifs.

«Soit un homme du XIX ${ }^{\mathrm{e}}$ siècle désireux d'améliorer les moyens de transport de l'époque. On lui avait bien appris que pour mettre au point de nouvelles techniques, il fallait commencer par bien se pénétrer des problèmes existants. Aussi s'était-il lancé dans une étude approfondie, comparant les diverses voitures à chevaux. II avait recherché soigneusement, par les méthodes les plus raffinées, comment variait la vitesse selon le dessin et la matière des divers types d'axes, selon les techniques de harnachement [...] » (id., p. 60) 
Papert conclut avec raison que cet homme n'a pu faire progresser les transports et que ce n'est pas l'étude (d'origine descriptive) détaillée d'un moyen de transport qui peut permettre la conception (usage prescriptif) de son successeur. II fait ensuite un parallèle avec la pédagogie, où il préconise d'inventer l'équivalent pédagogique de l'automobile plutôt que de décrire son ancêtre. Une description d'une technique (usage descriptif d'une méthode de conception, donc d'origine prescriptive) n'est de peu de secours pour le concepteur, hormis à des fins de rétro-ingénierie, comme nous l'avons montré plus haut.

Bereiter (en préparation) propose une autre parabole qui, elle, nous alerte sur les dangers possibles de concepteurs (prescriptifs) qui se refusent à valider leur produit de manière prescriptive, c'est-à-dire qui se refusent à adopter une démarche d'évaluation, au profit d'une démarche de vérification.

«Dans un magazine de 1908, apparut une publicité pour une automobile, la Réo [...]. Dans l'argumentaire il était indiqué que le volant était à sa place logique : au centre. Si le principe de la conception des automobiles avait été calqué sur celui de la recherche en éducation, on aurait consacré des années de recherches pour comparer les effets des différentes positions du volant. Le résultat probable aurait été qu'une position offre une meilleure visibilité selon un certain critère et qu'une autre en offre une meilleure selon un autre. II y aurait eu des débats méthodologiques [...] Aujourd'hui, il y aurait encore des partisans du volant à gauche, critiquant les partisans du volant à droite, et un nombre considérable de gens persuadés que le volant devrait être au milieu, "à sa place logique" ». (Bereiter, en préparation, non paginé)

Nous avons tenté de montrer ici qu'aucun des courants exposés précédemment ne peut être tenu responsable de ses utilisations abusives : la critique de la non-utilisabilité des méthodes prescriptives ne vaut pas plus que l'imposition de théories d'origine descriptive à des fins prescriptives. La solution à ce débat passerait sans doute par l'usage raisonné et cohérent de ces deux approches. Comme l'indique Sfard (1998, p. 10), «Lorsqu'une théorie est traduite en prescription d'enseignement, l'exclusivité devient le pire ennemi de la réussite. »

\section{Remerciements}

Nous remercions vivement Jacques Baillé, Pascal Bressoux et Benoît Lemaire pour leurs commentaires d'une version précédente de cet article. Les participants du symposium de juillet 2000 à Carcassonne intitulé «Les sciences de l'éducation, des 
recherches, une discipline » et dirigé par Jean-François Marcel sont également remerciés pour leurs commentaires à propos du cadre présenté dans cet article.

\section{REFERENCES BIBLIOGRAPHIQUES}

ALTET, M. (1994). - La formation professionnelle des enseignants. Paris : P.U.F.

ANDERSON, J. R., REDER, L. M., SIMON, H. A. (1995). Applications and misapplications of cognitive psychology to mathematics education. Pittsburgh : Université Carnegie Mellon.

BERBAUM, J. (1982). - Etude systémique des actions de formation. Paris : P.U.F.

BEREITER, C. (En préparation). - Education and mind in the knowledge age.

BERTRAND, Y. (1993). - Préface. In R. Viau (Ed.), La planification de l'enseignement, deux approches, deux visions ? Sherbrooke : CRP, p. 9-11.

BLOOM, B. S., ENGLEHART, M. D., FURST, E. J., HILL, W. H., KRATHWOHL, D. R. (1969). - Taxonomie des objectifs pédagogiques, Domaine cognitif. Montréal : Education Nouvelle.

BOBBITT, J. F. (1918). - The Curriculum. New York : Arno Press.

BONNER, J. (1988). - Implications of cognitive theory for instructional design : revisited. Educational Communication and Technology Journal, 36-1, p. 3-14.

BRADEN, R. A. (1996). - The case for linear instructional design and development : a commentary on models, challenges, and myths. Educational Technology, 36-2, p. 5-23.

BRU, M. (1991). - Les variations didactiques dans l'organisation des conditions d'apprentissage. Toulouse : Editions Universitaires du Sud.

BRU, M. (1994). - Quelles orientations pour les recherches sur la pratique de l'enseignement ? L'année de la Recherche en Sciences de l'Éducation, 1, p. 165-174.

BRU, M. (1998). - Qu'y a-t-il à prouver quand il s'agit d'éducation ? In C. Hadji, J. Baillé (Eds), Recherche et éducation, vers une «nouvelle alliance». Bruxelles : De Boeck, p. 45-66.

BRUNER, J. S. (1970). - Some theorems on instruction. In E. Stones (Ed.), Readings in Educational Psychology : Learning and Teaching. Londres : Methuen, p. 112-124.

BURTON, J. K., MOORE, D. M., MAGLIARO, S. G. (1996). - Behaviorism and Instructional Technology. In D. $\mathrm{H}$. Jonassen (Ed.), Handbook of Research for Educational Communications and Technology. New York : McMillan, p. 46-73.

CARLGREN, I. (1999). - Professionalism and teachers as designers. Journal of Curriculum Studies, 31-1, p. 43-56.

CARR, W. (1990). - Educational theory and its relation to educational practice. In N. Entwistle (Ed.), Handbook of Educational Ideas and Practices. Londres : Routledge, p. 100-109.

CLARK, C. M., DUNN, S. (1991). - Second-generation research on teachers' planning, intentions, and routines. In H. C. Waxman, H. J. Walberg (Eds), Effective teaching: Current research. Berkeley: McCutchan, p. 183-201.

CLARK, C. M., PETERSON, P. L. (1986). - Teachers' thought processes. In M.C. Wittrock (Ed.), Handbook of research on teaching. New York : Mac Millan, p. 255-296.

CLARK, R. E. (1983). - Reconsidering research on learning from media. Review of Educational Research, 53-4, p. 445-459.

CRAHAY, M. (1998). - Peut-on, et comment, concilier recherche en éducation et réflexion de l'action éducative ? In C. Hadji, J. Baillé (Eds), Recherche et éducation, vers une « nouvelle alliance ». Bruxelles : De Boeck, p. 125-160. 
D’HAINAUT, L. (1980). - La régulation dans les systèmes éducatifs. Paris : Unesco.

DALE, E. (1969). - Audio visual methods in teaching. New York : Holt, Rinehart and Winston.

DE CORTE, E., GeERligs, C. T., LAGeRWeiJ, N. A. J., PETERS, J. J., VANDENBERGHE, R. (1979). Les fondements de l'action didactique. Bruxelles : De Boeck.

DE KETELE, J.-M. (1986)(Ed.). - L'évaluation : approche descriptive ou prescriptive ? Bruxelles : De Boeck.

DE LANDSHEERE, G. (1994). - Le pilotage des systèmes d'éducation. Bruxelles : De Boeck.

DE LANDSHEERE, V., DE LANDSHEERE, G. (1984). - Définir les objectifs de l'éducation. Paris : P.U.F.

DE MONTMOLLIN, M. (1971). — L’enseignement programmé. Paris : P.U.F., Que sais-je ?

DERRY, S., LESGOLD, A. (1996). - Toward a situated social practice model for instructional design. In D. C. Berliner, R. C. Calfee (Eds), Handbook of Educational Psychology. New York : McMillan, p. 787-806.

DESSUS, P., LEMAIRE, B. (1999). - La technologie des médias joue-t-elle un rôle dans la transmission des connaissances ? In S. Agostinelli (Ed.), Comment penser la communication des connaissances ? Du CD-Rom à I'Internet. Paris : L'Harmattan, p. 253-264.

DICK, W. (1992). - An instructional designer's view of constructivism. In T. M. Duffy, D. H. Jonassen (Eds), Constructivism and the Technology of Instruction : a Conversation. Hillsdale : Erlbaum, p. 91-98.

DOYLE, W. (1986). - Paradigmes de recherche sur l'efficacité des enseignants. In M. Crahay, D. Lafontaine (Eds), L'art et la science de l'enseignement. Bruxelles : Labor, p. 435-481.

DUNKIN, M. J., BIDDLE, B. J. (1974). - The study of teaching. New York : Holt, Rinehart and Winston.

ERAUT, M. (1996). - Conceptual frameworks and historical development. In T. Plomp, D. P. Ely (Eds). International Encyclopedia of Educational Technology. Oxford : Pergamon, p. 1-17.

GRUBER, H., LAW, L.-C., MANDL, H., RENKL, A. (1995). - Situated learning and transfer. In P. Reimann, H. Spada (Eds), Learning in humans and machines. Oxford : Pergamon, p. 168-188.

JACKSON, P. W. (1996). - Conceptions of Curriculum and Curriculum specialists. In P. W. Jackson (Ed.), Handbook of Research on Curriculum. New York : MacMillan, p. 3-40.

KERR, S. T. (1981). - How teachers design their materials : Implications for instructional design. Instructional Science, 10, p. 363-378.

KIBLER, R. J., CEGALA, D. J., MILES, D. T., BARKER, L. L. (1974). - Objectives for Instruction and Evaluation. Washington : Département de l'enseignement Audiovisuel.

KLEIN, J. D. (1991). - Preservice teacher use of learning and instructional design principles. Educational Technology Research and Development, 39-3, p. 83-89.

KLIEBARD, H. M. (1995). - The Tyler rationale revisited. Journal of Curriculum Studies, 27-1, p. 81-88.

KOZMA, R. B. (1991). - Learning with media. Review of Educational Research, 61-2, p. $179-211$.

LEINHARDT, G., GREENO, J. G. (1986). - The cognitive skill of teaching. Journal of Educational Psychology, 78-2, p. 75-95.

LEVIE, W. H. (1989). - Media attributes. In M. Eraut (Ed.), International Encyclopedia of Educational Technology. Oxford : Pergamon, p. 398-401.

LOCKEE, B., BURTON, J. K., CROSS, L. H. (1999). - No comparison: Distance education finds a new use for 'No Significant Difference'. Educational Technology Research and Development, 47-3, p. 33-42.

MAGER, R. (1977). - Comment définir des objectifs pédagogiques. Paris : Bordas.

MERRILL, M. D., DRAKE, L., LACY, M. J., PRATT, J. (1996). - Reclaiming instructional design. Educational Technology, 36-5, p. 5-7. 
MONTAGUE, W. E. (1986). - Technical training and instructional systems development : Can a cognitive model help ? In T. G. Sticht, F. R. Chang, S. Wood (Eds), Advances in Reading/Language research, 4. Greenwich : JAI Press, p. 119-138.

PAPERT, S. (1981). - Jaillissement de l'esprit, ordinateurs et apprentissage. Paris : Flammarion.

PETRAGLIA, J. (1998). - The real world on a short leach : the (mis)application of constructivism to the design of educational technology. Educational Technology Research and Development, 46-3, p. 53-65.

PINKER, S. (2000). - Comment fonctionne l'esprit. Paris : Odile Jacob.

PROVENCHER, G. (1984). - L'évaluation de la qualité de l'apprentissage scolaire. In C. Paquette (Ed.), Des pratiques évaluatives. Ottawa : NHP, p. 95-128.

RIEBEN, L. (1988). - Un point de vue constructiviste sur la pédagogie de la maîtrise. In M. Huberman (Ed.), Assurer la réussite des apprentissages scolaires ? Neuchâtel : Delachaux et Niestlé, p. 127-154.

ROMISZOWSKI, A. J. (1996). - Systems approach to desing and development. In T. Plomp, D. P. Ely (Eds), International Encyclopedia of Educational Technology. Oxford : Pergamon, p. 37-43.

SAUVÉ, L. (1993). - La planification de l'enseignement en formation à distance. In R. Viau (Ed.), La planification de l'enseignement, deux approches, deux visions ? Sherbrooke : CRP, p. 63-90.

SFARD, A. (1998). - On two metaphors for learning and the dangers of choosing just one. Educational Researcher, 27-2, p. 4-13.

SHULMAN, L. S. (1986). - Paradigms and research programs in the study of teaching : a contemporary perspective. In M.C. Wittrock (Ed.), Handbook of Research on Teaching. New York : McMillan, p. 3-36.

SIMON, H. A. (1974). - La science des systèmes, science de l'artificiel. Paris : Epi.

SKINNER, B. F. (1968). - La révolution scientifique de l'enseignement. Bruxelles : Dessart.

TENNYSON, R. D. (1993). - Instructional design models and authoring activities. In J. M. Spector, M. C. Polson, D. J. Muraida (Eds), Automating instructional design. Englewood Cliffs : Educational technology publications, p. 330-338.

TROLLIP, S. R. (1996). - Courseware design. In T. Plomp, D. P. Ely (Eds), International Encyclopedia of Educational Technology. Oxford : Pergamon, p. 173-177.

TYLER, R. W. (1950). - Basic principles of curriculum and instruction. Chicago : Presses de l'Université de Chicago.

VIAU, R. (1993)(Ed.). - La planification de l'enseignement, deux approches, deux visions ? Québec : C.R.P.

WIBURG, K. M. (1995). - An historical perspective on instructional design : is it time to exchange Skinner's teaching machine for Dewey's toolbox ? Computer Support for Collaborative Learning (CSCL'95). Bloomington.

WILSON, B. G. (1995). - Maintaining the ties between learning theory and instructional design. Conférence Annuelle de l'American Educational Research Association. San Francisco.

YINGER, R. J. (1986). - Examining thought in action : a theoretical and methodological critique of research on interactive teaching. Teaching and Teacher Education, 2-3, p. 263-282.

YOUNG, M. F. (1993). - Instructional design for situated learning. Educational Technology Research and Development, 41-1, p. 43-58.

YOUNG, A. C., REISER, R. A., DICK, W. (1998). - Do superior teachers employ systematic instructional planning procedures ? A descriptive study. Educational Technology Research and Development, 46-2, p. $65-78$.

ZAHORIK, J. A. (1975). - Teacher's planning models. Educational Leadership, 33-2, p. 134-199. 
Philippe Dessus

Laboratoire des sciences de l'éducation, Université Pierre-Mendès-France et IUFM,

Grenoble

\section{NOTES}

(1) La planification de l'enseignement a surtout été étudiée dans ses aspects prescriptifs, à tel point qu'il serait difficile de recenser tous les modèles dévolus à ce processus (Braden, 1996, fait état de plus de soixante méthodes). II serait à peine plus aisé de répertorier les travaux proposant une classification de ces modèles (Clark \& Peterson, 1986).

(2) On peut même remonter aux années vingt avec Bobbitt (1918, cité notamment par Jackson, 1992), qui a produit le premier travail visant à concevoir un curriculum.

(3) La planification a été définie comme l'activité mise en œuvre hors de la présence des élèves, devant une « classe vide », définition qui contraint la planification dans un cadre strictement temporel. On a également soutenu que la planification est ce «[...] que les enseignants font quand ils disent qu'ils planifient » (Clark \& Dunn, 1991, p. 185), définition tautologique et infalsifiable.

(4) Toutefois, la nature descriptive originelle des taxonomies serait à vérifier de plus près que nous le permet l'ouvrage de Bloom et al. (1969). Leurs auteurs indiquent qu'ils ont « [...] procédé à l'inventaire des multiples objectifs proposés par les établissements scolaires et les revues spécialisées. » (id., p. 18) ce qui n'est pas une formulation très claire de leur méthode.

(5) Nous ne ferons pas référence, dans cet article, au «paradigme écologique », pourtant souvent cité comme l'un des courants majeurs de l'étude de l'enseignement. Les travaux qui s'en réclament nous paraissent difficilement classables dans l'un des courants présentés ici. De plus, la planification de l'enseignement y tient une place inexistante.

(6) La méthode de Tennyson (1993) met en œuvre une évaluation de faisabilité dans la phase de spécification, une évaluation formative dans la phase de conception, une évaluation sommative dans la phase de production.

(7) Pour faire un parallèle avec un autre domaine, signalons que de nombreux manuels de recherche en éducation prescrivent des étapes de la recherche qui ne correspondent pas à la manière dont les 
chercheurs en éducation réalisent leurs propres travaux. Ces manuels sont-ils pour autant inutiles ?

(8) Nous avons choisi de présenter le travail de Bru (1991) dans les approches cognitives car nous pouvons le rattacher au courant de recherche sur la pensée des enseignants.

(9) D'autres travaux (Kerr, 1981 ; Klein, 1991) adoptent la même méthode, si ce n'est que Kerr analyse les représentations des enseignants via des questionnaires, et que Klein vérifie que l'essentiel des méthodes prescrites a été acquis par les enseignants

(10) Le fait que l'on ait du mal à rattacher les pratiques effectives des enseignants à des méthodes couramment prescrites pourrait venir du fait que la procédure qu'ils suivent a été incorporée en routine, et ne leur est donc pas plus accessible qu'elle ne l'est aux chercheurs.

(11) Par exemple, équilibrer un compte en banque ne peut être considéré a priori comme une tâche authentique : cela peut être le cas pour des étudiants, mais pas pour des élèves de primaire (Petraglia, 1998). 